\title{
Physiological effects of a pollutant gradient - introduction
}

\author{
J. M. Capuzzo \\ Department of Biology, Woods Hole Oceanographic Institution, Woods Hole, Massachusetts 02543, USA
}

Physiological responses of marine organisms to pollutants are dependent on the bioavailability, uptake, accumulation and disposition of contaminants within the body, and on the interactive effects of multiple contaminants. In this regard, physiological responses are integrators of sub-cellular and cellular processes, and may be indicative of the overall fitness of the individual organism; they contribute also to our understanding of possible consequences of pollution to the population. The most important physiological changes associated with contaminant exposure are those that may adversely affect the organism's growth and survival and, thus, its ability to contribute to the population gene pool. Physiological indices linked to the survival and growth potential of the individual (such as the bioenergetic variables, feeding, digestion and respiration), or to the reproductive and developmental potential of the population (such as reproductive effort and larval viability) are therefore potentially most effective in assessing the effects of pollution gradients.

However, techniques appropriate to the measurement of the energy budget of an animal, i.e. rates of feeding, digestion, respiration and excretion, under physiological steady-state conditions, have been developed for relatively few organisms. For example, some suspension-feeding bivalve molluscs (such as the common mussel Mytilus edulis) have proved wellsuited to the laboratory determination of the so-called 'balanced energy equation', or scope for growth; they are sessile, do not suffer from prolonged handling stress, and it is technically straight-forward to estimate rates of feeding, which are virtually continuous, on suspended particulate material. Crabs Carcinus maenas on the other hand, are active predators, with very variable rates of respiration reflecting, in part, a discontinuous feeding cycle; to establish physiological steady-states for the reliable determination of energy balance in these circumstances is difficult, requiring many hours of continuous measurement to achieve integrated estimates of the appropriate rates. As a result, the bioenergetic approach to assessing the effects of pollutants has been limited in phylogenetic scope, although the principles involved should apply to a wide range of organisms.

At the GEEP Workshop the physiological energetics approach was applied to mussels Mytilus edulis from both field and mesocosm samples, and also to the periwinkle Littorina littorea. Acknowledging the variability so apparent in 'whole animal' physiology of crabs, respiration rates of isolated gill tissues were measured as a potential index of pollutant effect, and the same approach was adopted as a comparative exercise with the gill tissues of mussels. A standard bioassay technique was used to estimate the viability of embryos of mussels from the mesocosm experiment. Finally, a detailed analysis of the lipid composition of both mussel and crab tissue was carried out to provide a link between the cellular and physiological studies. 